Center for

Mathematical Economics

Working Papers

September 2014

On Repeated games with imperfect public monitoring: Characterization of Continuation payoff processes

Mathias Staudigl

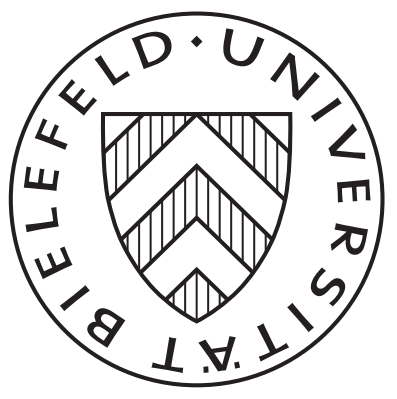




\title{
On Repeated games with imperfect public monitoring: Characterization of Continuation payoff processes*
}

\author{
Mathias Staudigl ${ }^{+}$
}

September 16, 2014

\begin{abstract}
This note contains complementary information to the paper Staudigl and Steg (2014). We present a martingale characterization of continuation payoff processes in a class of repeated games with imperfect public monitoring. Our martingale approach allows us to work out a clear connection between the discrete time and continuous time payoff processes. A general proof of convergence is the open issue in this literature, and I strongly belief that the characterization result reported here is the key to solve this problem.
\end{abstract}

Keywords: Repeated games, Public Perfect Equilibrium, Martingale Representation.

\section{Introduction}

In Staudigl and Steg (2014) we have shown that a recent class of repeated games in continuous-time can be motivated via a sequence of discrete-time repeated games. In that paper we prove the convergence of the repeated game dynamics and the total expected payoffs of the players. However, our analysis does not allow us to say anything about the relation between public perfect equilibria in the approximating discrete-time games and the limit game in continuous-time. This is, in general, a completely open question. The

${ }^{*}$ I thank Jan-Henrik Steg and Fabien Gensbittel for useful discussions on this topic. Financial support from the Vienna Science and Technology Fund (WWTF) under project fund MA 09-017 is gratefully acknowledged.

${ }^{\dagger}$ Center for Mathematical Economics, Bielefeld University, Germany. e-mail: mathias.staudigl@ uni-bielefeld.de; website: mwpweb.eu/MathiasStaudigl. 
purpose of this short note is to indicate a promising way to reduce the gap in our understanding of equilibrium payoff processes in discrete and continuous time. The exposition follows the notation of Staudigl and Steg (2014) and I refer to concepts introduced in that paper rather bluntly. However, in order to understand the material of this note it is not necessary to understand the convergence argument of the family of repeated games. It suffices to have a look at sections 2 and 3 of Staudigl and Steg (2014).

\section{Continuation payoffs in discrete-time}

Since perfect public equilibria are recursive, they can be characterized by dynamic programming methods as described in Abreu et al. (1990). Here we present an alternative explicit characterization of any continuation value process, which is the closest as possible to the corresponding continuous-time characterization presented in Section 3.

Following Staudigl and Steg (2014), we think of our family of discrete-time games $\Gamma^{h}$ as being played in real time, but only at regularly spaced time points $t_{n}^{h} \triangleq n h$ a change takes place. The discount factor in this game is given by $\delta^{h} \equiv \delta=e^{-r h}$, where $r>0$ is an exogenously given interest rate. Players observe a public signal process $X^{h}=\left\{X_{t_{n}^{h}}^{h} ; n \geq\right.$ $0\}$ and nothing else in the game. The information filtration of each player is hence given by $\mathcal{F}_{n}^{h} \triangleq \sigma\left(X_{t_{k}^{h}}^{h} ; 0 \leq k \leq n\right)$. A pure public strategy for player $i$ in this game is an $\left\{\mathcal{F}_{n}^{h}\right\}_{n \geq 0}$ adapted process $\alpha^{i, h}=\left\{\alpha_{n}^{i, h} ; n \geq 0\right\}$ with values in the finite set $A^{i}$. Given a pure public strategy profile $\alpha^{h}$ the repeated game dynamics is constructed on a common probability space and the players control the distribution of the signals. This "strategic measure" is denoted by $\mathbb{P}^{\alpha^{h}}$. An explicit construction of this measure is given in Section 3 of Staudigl and Steg (2014). Given a discrete-time pure public strategy $\alpha^{h}$, define the payoff processes

$$
\begin{aligned}
& U_{n}^{i, \alpha^{h}} \triangleq \mathrm{E}^{\mathbb{P}^{\alpha^{h}}}\left[\sum_{k=0}^{\infty} \delta^{k}(1-\delta) g^{i}\left(\alpha_{k}^{h}\right) \mid \mathcal{F}_{n}^{h}\right], \\
& W^{i, \alpha^{h}} \triangleq \mathrm{E}^{\mathbb{P}^{\alpha^{h}}}\left[\sum_{k \geq n} \delta^{k-n}(1-\delta) g^{i}\left(\alpha_{k}^{h}\right) \mid \mathcal{F}_{n}^{h}\right] .
\end{aligned}
$$

We refer to the process $W^{i, \alpha^{h}}=\left\{\left(W_{n}^{i, \alpha^{h}}, \mathcal{F}_{n}^{h}\right) ; n \in \mathbb{N}_{0}\right\}$ as the continuation value process of player $i . W_{n}^{i, \alpha^{h}}$ is the remaining utility of player $i$ under the public strategy profile $\alpha^{h}$ when the game reached period $n$ (summarized by the information structure $\mathcal{F}_{n}^{h}$ ). Observe that 
both processes are connected by the identity

$$
U_{n}^{i, \alpha^{h}}=\sum_{k=0}^{n-1} \delta^{k}(1-\delta) g^{i}\left(\alpha_{k}^{h}\right)+\delta^{n} W_{n}^{i, \alpha^{h}}
$$

The process $U^{i, \alpha^{h}}=\left\{\left(U_{n}^{i, \alpha^{h}}, \mathcal{F}_{n}^{h}\right) ; n \geq 0\right\}$ is a bounded martingale under the probability measure $\mathbb{P}^{\alpha^{h}}$. Set $U^{\alpha}=\left(U^{i, \alpha^{h}}\right)_{1 \leq i \leq N}$.

Our first representation result is a Doob-Decomposition of the continuation payoff process.

Proposition 2.1. A bounded $\left\{\mathcal{F}_{n}^{h}\right\}_{n \in \mathbb{N}_{0}}$-adapted process $\left\{W_{n}\right\}_{n \in \mathbb{N}_{0}}$ is the continuation value process induced by the public strategy profile $\alpha^{h}$ if and only if it solves the stochastic difference equation

$$
W_{n}=W_{0}+\frac{1-\delta}{\delta} \sum_{k=0}^{n-1}\left[W_{k}-g\left(\alpha_{k}^{h}\right)\right]+\sum_{k=0}^{n-1} d_{k+1}^{h}
$$

where

$$
d_{n+1}^{h} \triangleq W_{n+1}-\mathrm{E}^{\mathbb{P}^{\alpha^{h}}}\left[W_{n+1} \mid \mathcal{F}_{n}^{h}\right] .
$$

Proof. For ease of notation, we omit the mesh size $h$ in the proof. For every discrete time process $\left(W_{n}\right)_{\mathbb{N}_{0}}$ that is integrable under the measure $\mathbb{P}^{\alpha}$ we can define a Doob decomposition $W=M+N$ into a martingale and a predictable process. The martingale increments are $\Delta M_{n-1}=d_{n}$ given in (3). Hence, $W$ solves (2) iff the first sum is that of the increments $\Delta N_{k}=\mathrm{E}^{\mathbb{P}^{\alpha}}\left[\Delta W_{k} \mid \mathcal{F}_{k}\right]$, which is equivalent to

$$
W_{n}=(1-\delta) g\left(\alpha_{n}\right)+\delta \mathrm{E}^{\mathbb{P}^{\alpha}}\left[W_{n+1} \mid \mathcal{F}_{n}\right], \quad n \in \mathbb{N}_{0} .
$$

It is trivial to see that $W^{\alpha}=\left\{\left(W_{n}^{\alpha}, \mathcal{F}_{n}\right) ; n \in \mathbb{N}_{0}\right\}$ satisfies this recursion. If another bounded process $W$ satisfies it as well, then by iteration

$$
W_{n}=\mathrm{E}^{\mathbb{P}^{\alpha}}\left[(1-\delta) \sum_{l=0}^{k-1} \delta^{l} g\left(\alpha_{n+l}\right)+\delta^{k} W_{n+k} \mid \mathcal{F}_{n}\right]
$$

for any $k \geq 0$. Taking the limit $k \rightarrow \infty$, we obtain $W_{n}^{\alpha}$ on the right-hand side by dominated convergence, as $W$ is bounded (it must be inside the convex compact set $\mathcal{V}=\operatorname{conv}[g(\mathcal{A})]$ ).

Our goal in this section is to decompose the bounded martingale $U^{\alpha^{h}}$ orthogonally 
into a stochastic integral and a square integrable martingale part. The motivation for this is that in continuous time the continuation payoff process is the solution to a BSDE driven by a Wiener process (which will be introduced in Section 3). To obtain an explicit expression for the integrand of the discrete approximation of the Wiener process, a further martingale decomposition is needed. To perform such a decomposition we first have to identify the driving noise process under the probability measure $\mathbb{P}^{\alpha^{h}}$. To this end, consider the martingale

$$
B_{n}^{\alpha^{h}} \triangleq \bar{B}_{t_{n}^{h}}^{h} \quad \forall n \geq 0
$$

where $\bar{B}^{h}$ is the right-continuous process defined in eq. (16) of Staudigl and Steg (2014). Following the arguments in that paper, we can alternatively write ${ }^{1}$

$$
B_{n}^{\alpha^{h}}=B_{n}^{h}-h \sum_{k=0}^{n-1} \mu\left(\alpha_{k}^{h}\right)
$$

so that

$$
\Delta B_{n}^{\alpha^{h}} \triangleq B_{n+1}^{\alpha^{h}}-B_{n}^{\alpha^{h}}=B_{n+1}-B_{n}^{h}-h \mu\left(\alpha_{n}^{h}\right)=\Delta B_{n}^{h}-h \mu\left(\alpha_{n}^{h}\right) .
$$

The Kunita-Watanabe decomposition (see Kunita and Watanabe (1967) or Jacod (1979), ch. IV, sec.2) of the bounded martingale $U^{\alpha^{h}}$ with respect to the martingale $B^{\alpha^{h}}$ is given by

$$
U_{n}^{\alpha^{h}}=U_{0}^{\alpha^{h}}+\sum_{k=0}^{n-1} \Phi_{k+1}^{h} \Delta B_{k}^{\alpha^{h}}+L_{n}^{h}
$$

where $L^{h}$ is a bounded martingale with $L_{0}^{h}=\mathbf{0} \in \mathbb{R}^{N}$ and strongly orthogonal to $B^{\alpha^{h}}$. This means that

$$
\begin{aligned}
& \mathrm{E}^{\mathbb{P}^{\alpha^{h}}}\left[\Delta L_{n}^{h} \mid \mathcal{F}_{n}^{h}\right]=0 \quad \forall n \geq 0, \text { and } \\
& \mathrm{E}^{\mathbb{P}^{\alpha^{h}}}\left[\Delta L_{n}^{i, h} \Delta B_{j, n}^{\alpha^{h}} \mid \mathcal{F}_{n}^{h}\right]=0 \quad \forall 1 \leq i \leq N, 1 \leq j \leq d, n \geq 0 .
\end{aligned}
$$

The process $\Phi^{h}=\left\{\Phi_{n}^{h} ; n \geq 1\right\}$ is an $\left\{\mathcal{F}_{n}^{h}\right\}_{n}$-predictable process taking values in $\mathbb{R}^{N \times d}$.

\footnotetext{
${ }^{1}$ Specifically $B_{n}^{h}=\sqrt{h} \sum_{k=0}^{n-1} \xi_{k+1}$.
} 
Observe that eq. (8) also implies that

$$
\mathrm{E}^{\mathbb{P}^{\alpha^{h}}}\left[\Delta L_{n}^{i, h} \Delta B_{j, n}^{h} \mid \mathcal{F}_{n}^{h}\right]=0 \quad \forall 1 \leq i \leq N, 1 \leq j \leq d, n \geq 0
$$

However, the process $B^{h}$ is not a martingale under the "strategic" measure $\mathbb{P}^{\alpha^{h}}$ (but it is one under the reference measure $\mathbb{P}=\rho^{\otimes \mathbb{N}}$, again see section 3 of Staudigl and Steg (2014) for the details). Taking first order differences in eq. (6) we see that

$$
\begin{aligned}
U_{n+1}^{\alpha^{h}}-U_{n}^{\alpha^{h}} & =\Phi_{n+1}^{h} \Delta B_{n}^{h}+\Delta L_{n}^{h}-h \Phi_{n+1}^{h} \mu\left(\alpha_{n}^{h}\right) \quad \mid \text { by eq. (5) } \\
& =\delta^{n}\left\{(1-\delta) g\left(\alpha_{n}^{h}\right)+\delta W_{n+1}^{h}-W_{n}^{h}\right\} \quad \mid \text { by eq. (1). }
\end{aligned}
$$

Hence,

$$
\delta\left[W_{n+1}^{h}-W_{n}^{h}\right]=(1-\delta)\left[W_{n}^{h}-g\left(\alpha_{n}^{h}\right)\right]-h \delta^{-n} \Phi_{n+1}^{h} \mu\left(\alpha_{n}^{h}\right)+\delta^{-n} \Delta L_{n}^{h}+\delta^{-n} \Phi_{n+1}^{h} \Delta B_{n}^{h}
$$

which, after dividing through $\delta$ and some rearrangements, gives

$$
W_{n+1}^{h}-W_{n}^{h}=\frac{1-\delta}{\delta}\left[W_{n}^{h}-g\left(\alpha_{n}^{h}\right)-\frac{h \delta^{-n}}{1-\delta} \Phi_{n+1}^{h} \mu\left(\alpha_{n}^{h}\right)\right]+\delta^{-(n+1)} \Phi_{n+1}^{h} \Delta B_{n}^{h}+\delta^{-(n+1)} \Delta L_{n}^{h} .
$$

Setting

$$
Z_{n}^{h}=\frac{1}{r} \delta^{-n} \Phi_{n}^{h} \quad \forall n \geq 1
$$

we get

$$
W_{n+1}^{h}-W_{n}^{h}=\frac{1-\delta}{\delta}\left[W_{n}^{h}-g\left(\alpha_{n}^{h}\right)-\frac{r h \delta}{1-\delta} Z_{n+1}^{h} \mu\left(\alpha_{n}^{h}\right)\right]+r Z_{n+1}^{h} \Delta B_{n}^{h}+\delta^{-(n+1)} \Delta L_{n}^{h} .
$$

The next steps are very familiar from the recursive approach to repeated games. Define the auxiliary one-shot game

$$
G^{h}(a, z) \triangleq g(a)+\frac{r h \delta}{1-\delta} z \mu(a)
$$

so that $G^{h}(\cdot, z): A \triangleq \prod_{1 \leq i \leq N} A^{i} \rightarrow \mathbb{R}^{N}$ is the auxiliary one-shot game with payoff sensitivity $z$ (the use of this terminology will become clear soon).

Lemma 2.2. Let $G: A \times \mathbb{R}^{N \times d} \rightarrow \mathbb{R}^{N}$ be defined as

$$
G(a, z)=g(a)+z \mu(a)
$$


Then $G^{h} \rightarrow$ G uniformly as $h \rightarrow 0^{+}$.

Then, we finally arrive at the representation

$$
W_{n+1}^{h}-W_{n}^{h}=\frac{1-\delta}{\delta}\left[W_{n}^{h}-G^{h}\left(\alpha_{n}^{h}, Z_{n+1}^{h}\right)\right]+r Z_{n+1}^{h} \Delta B_{n}^{h}+\delta^{-(n+1)} \Delta L_{n}^{h} .
$$

It is important to keep in mind that $Z^{h}=\left\{Z_{n}^{h} ; n \geq 1\right\}$ is $\left\{\mathcal{F}_{n}^{h}\right\}_{n \in \mathbb{N}_{0}}$-predictable. Conversely, for a fixed public strategy profile $\alpha^{h}$, consider a triple $\left(W^{h}, Z^{h}, L^{h}\right)$ satisfying eq. (11), where

- $Z^{h}=\left\{Z_{n}^{h} ; n \geq 1\right\}$ is $\left\{\mathcal{F}_{n}^{h}\right\}_{n \in \mathbb{N}_{0}}$-predictable,

- $L^{h}$ is square integrable martingale in $\mathbb{R}^{N}$ strongly orthogonal to $B^{\alpha^{h}}$ under $\mathbb{P}^{\alpha^{h}}$ starting at $\mathbf{0}$, and

- $W^{h}$ is a bounded adapted process living in $\mathcal{V} \triangleq \operatorname{conv}(g(A))$.

Then it is easy to see that $W^{h}$ is the continuation payoff process under the strategy profile $\alpha^{h}$. Indeed, under the just spelled out conditions satisfied by the triple $\left(W^{h}, Z^{h}, L^{h}\right)$, we get

$$
\begin{aligned}
W_{n}^{h} & =(1-\delta) g\left(\alpha_{n}^{h}\right)-r \delta Z_{n+1}^{h}\left(\Delta B_{n}^{h}-h \mu\left(\alpha_{n}^{h}\right)\right)-\delta^{-n} \Delta L_{n}^{h}+\delta W_{n+1}^{h} \\
& =(1-\delta) g\left(\alpha_{n}^{h}\right)-r \delta Z_{n+1}^{h} \Delta B_{n}^{\alpha^{h}}-\delta^{-n} \Delta L_{n}^{h}+\delta W_{n+1}^{h} .
\end{aligned}
$$

Iterating up $m \geq 0$ times gives

$$
W_{n}^{h}=\sum_{k=0}^{m-1}(1-\delta) \delta^{k-n} g\left(\alpha_{n+k}^{h}\right)-\sum_{k=0}^{m-1} r \delta^{k+1} Z_{n+k+1}^{h} \Delta B_{n+k}^{\alpha^{h}}-\delta^{-n}\left(L_{n+m}^{h}-L_{n}^{h}\right)+\delta^{m} W_{n+m}^{h} .
$$

Applying the operator $\mathrm{E}^{\mathbb{P}^{\alpha^{h}}}\left[\cdot \mid \mathcal{F}_{n}^{h}\right]$ to both sides of this equation gives for $N=n+m, m \geq$ 0 , the expression

$$
W_{n}^{h}=\mathrm{E}^{\mathbb{P}^{\alpha^{h}}}\left[\sum_{k=n}^{N-1}(1-\delta) \delta^{k-n} g\left(\alpha_{k}^{h}\right)+\delta^{N-n} W_{N}^{h} \mid \mathcal{F}_{n}^{h}\right] .
$$

Using the boundedness of the process $W^{h}$ allows us to pass to $N \rightarrow \infty$ using the dominated convergence theorem. This shows that

$$
W_{n}^{h}=\mathrm{E}^{\mathbb{P}^{\alpha^{h}}}\left[\sum_{k=n}^{\infty}(1-\delta) \delta^{k-n} g\left(\alpha_{k}^{h}\right) \mid \mathcal{F}_{n}^{h}\right]=W_{n}^{\alpha^{h}} \quad \mathbb{P}^{\alpha^{h}}-\text { a.s. }
$$


Hence, we have show the following characterization result.

Theorem 2.3. An adapted process $W^{h}=\left\{W_{n}^{h} \mathcal{F}_{n}^{h} ; n \geq 0\right\}$ living in $\mathcal{V}$ is the continuation value process under the public strategy profile $\alpha^{h}$ if and only if there are processes $\left(Z^{h}, L^{h}\right)$ satisfying the following conditions:

- $Z^{h}=\left\{Z_{n}^{h} ; n \geq 1\right\}$ is a $\left\{\mathcal{F}_{n}^{h}\right\}_{n \geq 0}$-predictable process taking values in $\mathbb{R}^{N \times d}$;

- $L^{h}=\left\{L_{n}^{h} ; n \geq 0\right\}$ is a square integrable martingale in $\mathbb{R}^{N}$ with $L_{0}^{h}=\mathbf{0}$ which is strongly orthogonal to $B^{\alpha^{h}}$ under $\mathbb{P}^{\alpha^{h}}$;

- The sums

$$
\sum_{n \geq 0} r Z_{n+1}^{h} \Delta B_{n}^{\alpha^{h}}+\sum_{n \geq 0} \delta^{-n} \Delta L_{n}^{h}
$$

exist in $L^{2}\left(\mathbb{P}^{\alpha^{h}}\right)$, and

- The triple $\left(W^{h}, Z^{h}, L^{h}\right)$ satisfies the difference equation (11).

Remark 2.4. In Staudigl and Steg (2014) we construct the measure $\mathbb{P}^{\alpha^{h}}$ to be absolutely continuous with respect to the reference measure $\mathbb{P}$. Hence, it is immaterial whether we impose the transversality condition with respect to the strategic measure $\mathbb{P}^{\alpha^{h}}$ or $\mathbb{P}$.

The above theorem shows that, given a public strategy profile $\alpha^{h}$, the solution of the repeated game dynamic is a triple of processes $\left(W^{h}, Z^{h}, L^{h}\right)$. The next result gives us explicit expressions for the coefficients in the characterizing difference equation of the continuation payoff process.

Proposition 2.5. For all $h>0$ and $n \geq 0$ we have

$$
r h Z_{n+1}^{h}=\mathrm{E}^{\mathbb{P}^{\alpha^{h}}}\left[\left(W_{n+1}^{h}-W_{n}^{h}\right)\left(B_{n+1}^{\alpha^{h}}-B_{n}^{\alpha^{h}}\right)^{\top} \mid \mathcal{F}_{n}^{h}\right]
$$

and

$$
L_{n+1}^{h}-L_{n}^{h}=\delta^{n+1}\left(d_{n+1}^{h}-r Z_{n+1}^{h} \Delta B_{n}^{\alpha^{h}}\right),
$$

where $d^{h}$ is the martingale difference sequence defined in (3).

Proof. From the previous arguments we know that

$$
\delta W_{n+1}^{h}-W_{n}^{h}=r \delta Z_{n+1}^{h} \Delta B_{n}^{\alpha^{h}}+\delta^{-n} \Delta L_{n}^{h}-(1-\delta) g\left(\alpha_{n}^{h}\right)
$$


Multiplying both sides by $\left(\Delta B_{n}^{\alpha^{h}}\right)^{\top}$ and applying the operator $\mathrm{E}^{\mathbb{P}^{a^{h}}}\left[\cdot \mid \mathcal{F}_{n}^{h}\right]$ we get

$$
\mathrm{E}^{\mathbb{P}^{\alpha^{h}}}\left[\left(\delta W_{n+1}^{h}-W_{n}^{h}\right)\left(B_{n+1}^{\alpha^{h}}-B_{n}^{\alpha^{h}}\right)^{\top} \mid \mathcal{F}_{n}^{h}\right]=r h \delta Z_{n+1}^{h} .
$$

Here we used the fact that the sensitivity process $Z_{n+1}^{h}$ is $\mathcal{F}_{n}^{h}$-predictable. Since $W_{n}^{h}$ is $\mathcal{F}_{n}^{h}$-measurable, this is equal to

$$
\mathrm{E}^{\mathbb{P}^{\alpha^{h}}}\left[W_{n+1}^{h}\left(B_{n+1}^{\alpha^{h}}-B_{n}^{\alpha^{h}}\right)^{\top} \mid \mathcal{F}_{n}^{h}\right]=r h Z_{n+1}^{h},
$$

which in turn is equal to

$$
\mathrm{E}^{\mathbb{P}^{\alpha^{h}}}\left[\left(W_{n+1}^{h}-W_{n}^{h}\right)\left(B_{n+1}^{\alpha^{h}}-B_{n}^{\alpha^{h}}\right)^{\top} \mid \mathcal{F}_{n}^{h}\right]=r h Z_{n+1}^{h} .
$$

For the second representation, first recall the Doob-decomposition from Proposition 2.1:

$$
\delta W_{n+1}^{h}-W_{n}^{h}=\delta d_{n+1}^{h}-(1-\delta) g\left(\alpha_{n}^{h}\right)
$$

Combining this with the previous display we see immediately that

$$
\delta^{-n} \Delta L_{n}^{h}=\delta\left(d_{n+1}^{h}-r Z_{n+1}^{h} \Delta B_{n}^{\alpha^{h}}\right) .
$$

Note that these characterizations are general, as we have not imposed any equilibrium conditions (i.e. incentive compatibility). The last proposition shows that once $W^{h}$ and $Z^{h}$ are known, then $L^{h}$ is determined as a residual via eq. (13). In order to close the system of equations we need to formulate appropriate incentive compatibility conditions.

From the derivations presented in the next section, it will become clear that equation (11) is indeed the discrete-time analogue of the continuous-time representation theorem, first reported in Sannikov (2007). The derivation is just slightly more complicated because we do not have the predictable representation property of $L^{2}$-martingales w.r.t. the driving martingale $B^{\alpha^{h}}$ in general. ${ }^{2}$ We close this section with the following

\footnotetext{
${ }^{2}$ In discrete-time, it is well known that the only signal process which has the predictable representation property is the binary tree model. Assuming this simple structure in the discrete-time game might be fine for computational purposes (as in Cox et al. (1979)), it is arguably not very satisfactory from a theoretical point of view in games.
} 


\section{Continuation payoff process in continuous-time}

We now turn our attention to the continuous-time limit game. The 2-player version is due to Sannikov (2007). We here provide the relevant extensions to the $\mathrm{N}$-player case using the weak solution approach to optimal control. As in discrete-time, key to the derivation is the construction of continuation payoff processes. Given a public strategy profile $\alpha \in \mathcal{A}$ in the continuous-time game (see Staudigl and Steg (2014) for the relevant definition), let

$$
\begin{aligned}
& U_{t}^{i, \alpha}=\mathrm{E}^{\mathrm{P}^{\alpha}}\left[\int_{0}^{\infty} r e^{-r s} g^{i}\left(\alpha_{s}\right) \mathrm{d} s \mid \mathcal{W}_{t}\right], \text { and } \\
& W_{t}^{i, \alpha}=\mathrm{E}^{\mathrm{P}^{\alpha}}\left[\int_{t}^{\infty} r e^{-r(s-t)} g^{i}\left(\alpha_{s}\right) \mathrm{d} s \mid \mathcal{W}_{t}\right] .
\end{aligned}
$$

As in discrete-time, these processes are connected via the identity

$$
U_{t}^{\alpha}=\int_{0}^{t} r e^{-r s} g\left(\alpha_{s}\right) \mathrm{d} s+e^{-r t} W_{t}^{\alpha} .
$$

Under the probability measure $\mathrm{P}^{\alpha}$, the process $U^{\alpha}=\left\{U_{t}^{i, \alpha} ; t \geq 0\right\}$ is a bounded martingale with respect to the filtration $\mathcal{W}_{t}$, constructed in Staudigl and Steg (2014). On the resulting stochastic basis $\left(\mathbf{W}^{d+1}, \mathcal{W}^{d+1},\left\{\mathcal{W}_{t}\right\}_{t \geq 0}, \mathrm{P}^{\alpha}\right)$ the driving Brownian motion of the repeated game dynamics is the process $B^{\alpha}$, defined in Section 2 of Staudigl and Steg (2014). However, the filtration $\left\{\mathcal{W}_{t}\right\}_{t \geq 0}$ is bigger than the filtration generated by $B^{\alpha}$. Hence, the Itô representation theorem of $L^{2}$-martingales is not applicable. The necessary technical result we need for representing the martingale $U^{\alpha}$ in terms of an Itô-integral with respect to the Brownian motion $B^{\alpha}$ is the following classical fact.

Lemma 3.1 (Fujisaki et al. (1972)). Fix a public strategy $\alpha \in \mathcal{A}$. For every $\xi \in L^{2}\left(\mathcal{W}_{\infty}, \mathrm{P}^{\alpha} ; \mathbb{R}^{N}\right)$ there exist unique $\left\{\mathcal{W}_{t}\right\}_{t \geq 0}$-progressively measurable $\mathbb{R}^{N \times(d+1)}$-valued processes

$$
\Phi_{t}^{\alpha}=\left[\begin{array}{c}
\Phi_{t}^{1, \alpha} \\
\vdots \\
\Phi_{t}^{N, \alpha}
\end{array}\right], \text { where } \Phi_{t}^{i, \alpha}=\left[\Phi_{0}^{i, \alpha}(t), \ldots, \Phi_{d}^{i, \alpha}(t)\right] \quad 1 \leq i \leq N
$$

such that

$$
\mathrm{E}^{\mathrm{P}^{\alpha}}\left[\int_{0}^{\infty}\left\|\Phi_{s}^{\alpha}\right\|^{2} \mathrm{~d} s\right]<\infty \text {, where }\|z\|^{2}=\operatorname{tr}\left(z z^{\top}\right),
$$


and

$$
\xi^{i}=\mathrm{E}^{\mathrm{P}^{\alpha}}\left[\xi^{i}\right]+\int_{0}^{\infty} \sum_{k=0}^{d} \Phi_{k}^{i, \alpha}(t) \mathrm{d} B_{k}^{\alpha}(t) \quad 1 \leq i \leq N,
$$

where $B^{\alpha}$ is a $\mathrm{P}^{\alpha}$ Brownian motion (see Section 2 of Staudigl and Steg (2014)).

Proof. See proposition 2.31 in Bain and Crisan (2000).

Using this result, we are able to state and prove a nice representation formula satisfied by the continuation payoff process.

Theorem 3.2. For every public strategy profile $\alpha \in \mathcal{A}$, the continuation payoff process is the almost sure unique adapted process $W=\left\{\left(W_{t}, \mathcal{W}_{t}\right) ; t \geq 0\right\}$, satisfying the following conditions:

(i) $W_{t} \in \mathcal{V}=\operatorname{conv}[g(\mathcal{A})]$ a.s.-Leb $\times \mathrm{P}$, so that the transversality condition

$$
\lim _{t \rightarrow \infty} e^{-r t} \mathrm{E}^{\mathrm{P}}\left[\left\|W_{t}\right\|^{2}\right]=0
$$

is satisfied.

(ii) there is an $\mathbb{R}^{N \times d}$-valued progressive process $Z=\left\{Z_{t} ; t \geq 0\right\}$ in $L^{2}\left(\mathrm{P}^{\alpha}\right)$ and a square integrable process $\eta$ orthogonal to $B_{(1)}$, such that the pair $(W, Z)$ solves the stochastic integral equation

$$
W_{t}=\int_{t}^{\infty} r e^{-r(s-t)}\left[g\left(\alpha_{s}\right)+Z_{s} \mu\left(\alpha_{s}\right)\right] \mathrm{d} s-\int_{t}^{\infty} r e^{-r(s-t)} Z_{s} \mathrm{~d} B_{(1)}(s)+\eta_{t} .
$$

Proof. (i) is an obvious necessary condition for $W$ to be a continuation payoff process. The transversality condition is then a direct implication of the almost sure boundedness of the process.

(ii) For any public strategy profile $\alpha \in \mathcal{A}$, the martingale $U^{\alpha}=\left\{U_{t}^{\alpha}, \mathcal{W}_{t} ; t \geq 0\right\}$ is bounded. By the martingale convergence theorem there exists a random variable $U_{\infty} \in$ $L_{\mathcal{W}_{\infty}}^{2}\left(\mathrm{P}^{\alpha}\right)$ such that $\lim _{t \rightarrow \infty} U_{t}^{\alpha}=U_{\infty} \mathrm{P}^{\alpha}$-almost surely, and moreover

$$
\mathrm{E}^{\mathrm{P}^{\alpha}}\left[U_{\infty} \mid \mathcal{W}_{t}\right]=U_{t}^{\alpha} \quad \forall t \geq 0
$$

Using Lemma 3.1, there exists a $\left\{\mathcal{W}_{t}\right\}_{t \geq 0}$-progressively measurable process $\Phi^{\alpha}=\left\{\Phi_{t}^{\alpha} ; t \geq\right.$ $0\}$ such that

$$
U_{\infty}=\mathrm{E}^{\mathrm{P}^{\alpha}}\left[U_{\infty}\right]+\int_{0}^{\infty} \Phi_{s}^{\alpha} \mathrm{d} B_{s}^{\alpha}
$$


Taking condition expectations on both sides, we conclude

$$
U_{t}^{\alpha}=\mathrm{E}^{\mathrm{P}^{\alpha}}\left[U_{\infty}\right]+\int_{0}^{t} \Phi_{s}^{\alpha} \mathrm{d} B_{s}^{\alpha}=\int_{0}^{t} r e^{-r s} g\left(\alpha_{s}\right) \mathrm{d} s+e^{-r t} W_{t}^{\alpha} .
$$

Hence,

$$
e^{-r t} W_{t}^{\alpha}=\mathrm{E}^{\mathrm{P}^{\alpha}}\left[U_{\infty}\right]+\int_{0}^{t} \Phi_{s}^{\alpha} \mathrm{d} B_{s}^{\alpha}-\int_{0}^{t} r e^{-r s} g\left(\alpha_{s}\right) \mathrm{d} s .
$$

By Itô's formula, one sees

$$
\mathrm{d} W_{t}=r\left[W_{t}-g\left(\alpha_{t}\right)\right] \mathrm{d} t+e^{r t} \Phi_{t}^{\alpha} \mathrm{d} B_{t}^{\alpha} .
$$

Setting

$$
\Psi_{t}^{\alpha} \triangleq \frac{1}{r} e^{r t} \Phi_{t}^{\alpha} \quad \forall t \geq 0,
$$

and using the relation

$$
\mathrm{d} B_{t}^{\alpha}=\mathrm{d} B_{t}-f\left(\alpha_{t}\right) \mathrm{d} t, f(a)=(0, \mu(a))^{\top},
$$

we get

$$
\mathrm{d} W_{t}=r\left[W_{t}-g\left(\alpha_{t}\right)-\Psi_{t}^{\alpha} f\left(\alpha_{t}\right)\right] \mathrm{d} t+r \Psi_{t}^{\alpha} \mathrm{d} B_{t}
$$

Recall from Staudigl and Steg (2014) that $B=\left(B_{0}, B_{(1)}\right)$ is a partitioned vector of Brownian motions. Accordingly we partition the matrix-valued random variable $\Psi_{t}^{\alpha}$ as

$$
\Psi_{t}^{\alpha}=\left[\psi_{0}(t): Z_{t}\right]
$$

with $\psi_{0}(t)=\left(\psi_{0}^{1}(t), \ldots, \psi_{0}^{N}(t)\right)^{\top}$, and $Z_{t}=\left[Z_{k}^{i}(t)\right]_{1 \leq i \leq N ; 1 \leq k \leq d}$ and $N \times d$ matrix valued process. From this, it is immediate that

$$
\mathrm{d} W_{t}=r\left[W_{t}-g\left(\alpha_{t}\right)-Z_{t} \mu\left(\alpha_{t}\right)\right] \mathrm{d} t+r Z_{t} \mathrm{~d} B_{(1)}(t)+r \psi_{0}(t) \mathrm{d} B_{0}(t)
$$

Integrating from $t$ forward by exploiting the transversality condition (16), and setting $\eta_{t}^{\alpha}=e_{\infty}^{\alpha}-e_{t}^{\alpha}$, where

$$
e_{t}^{\alpha} \triangleq \int_{0}^{t} \psi_{0}(s) \mathrm{d} B_{0}(s)
$$


gives the representation (17).

Remark 3.3.

- In the statement of the Theorem, both processes $Z$ and $\eta$ depend on the public strategy $\alpha$. Difference strategies will lead to different coefficients.

- The random variable $e_{\infty}^{\alpha}$ appearing in the proof of the characterization theorem is well defined, thanks to the square integrability of the Brownian motion integrands. See also Protter (2005). Note that the process $e^{\alpha}=\left\{\left(e_{t}^{\alpha}, \mathcal{W}_{t}\right) ; t \geq 0\right\}$ is a P-martingale, strongly orthogonal to $B_{(1)}$.

\subsection{Equilibrium characterization}

The power of Theorem 3.2 is that it introducs a BSDE which must be solved by the continuation payoff process $W$ and corresponding martingale integrand $Z$ for a given public strategy profile $\alpha$. The next result is essentially a comparison principle for solutions of the BSDE (17), which allows us to derive precise conditions for a perfect public equilibrium payoff process. ${ }^{3}$ The key to this characterization is the auxiliary "one-shot" game

$$
G(a, z) \triangleq g(a)+z \mu(a) .
$$

From Lemma 2.2 we know that this is the uniform limit of the sequence of discrete-time auxiliary one-shot games $\left\{G^{h} ; h \in(0,1)\right\}$. In general, for any normal form game $u: A \rightarrow$ $\mathbb{R}^{N}$, we let $\operatorname{NE}(u)$ denote the pure-strategy Nash equilibria of $u$ (possibly empty). The definition of public equilibrium uses the following version of sequential rationality:

Definition 3.4. A public strategy profile $\alpha^{*} \in \mathcal{A}$ is a perfect public equilibrium if

$$
W_{t}^{i, \alpha^{*}} \geq W_{t}^{i,\left(\alpha^{i}, \alpha^{-i, *}\right)} \quad \forall t \geq 0, \mathrm{P}-\text { a.s. }
$$

for all players $i=1,2, \ldots, N$ and alternative strategies $\alpha^{i} \in \mathcal{A}^{i}$.

The next theorem shows that the characterization of public equilibria in the continuoustime game is close to what is known in discrete-time as the recursive approach.

\footnotetext{
${ }^{3}$ Observe that the BSDE (17) is high dimension, so the existence of a comparison principle is not immediate.
} 
Theorem 3.5. Suppose that the pair of adapted processes $(W, Z)$ solves the BSDE (17) for a given public strategy profile $\alpha \in \mathcal{A}$. Then $\alpha$ is a perfect public equilibrium strategy if and only if

$$
\alpha_{t} \in \operatorname{NE}\left(G\left(\cdot, Z_{t}\right)\right)
$$

for almost all $t$ and $\mathrm{P}-$-a.s.

Proof. (i) Suppose that the strategy profile $\alpha^{*}$ satisfies condition (19). We will show that this implies that $\alpha^{*}$ is a perfect public equilibrium strategy. For this it is enough to show that no single player has a profitable deviation from $\alpha^{*}$. Hence, let $\alpha^{i} \in \mathcal{A}^{i}$ be an arbitrary alternative public strategy of player $i$ and denote by $\alpha=\left(\alpha^{i}, \alpha^{-i, *}\right)$ the resulting deviation strategy profile. By definition,

$$
W_{t}^{\alpha}=\mathrm{E}^{\mathrm{P}^{\alpha}}\left[\int_{t}^{\infty} r e^{-r(s-t)} g\left(\alpha_{s}^{i}, \alpha_{s}^{-i, *}\right) \mathrm{d} s \mid \mathcal{W}_{t}\right] .
$$

Following Theorem 3.2, the continuation payoff process under strategy $\alpha^{*}$ is given by

$$
W_{t}^{\alpha^{*}}=\int_{t}^{\infty} r e^{-r(s-t)} g\left(\alpha_{s}^{*}\right) \mathrm{d} s-\int_{t}^{\infty} r e^{-r(s-t)} \Psi_{s}^{\alpha^{*}} \mathrm{~d} B_{s}^{\alpha^{*}},
$$

where $\Psi^{\alpha^{*}}=\left[\psi_{0}^{\alpha^{*}}: Z^{\alpha^{*}}\right]$. Since

$$
\begin{aligned}
0 & =W_{t}^{\alpha^{*}}-\int_{t}^{\infty} r e^{-r(s-t)} g\left(\alpha_{s}^{*}\right) \mathrm{d} s+\int_{t}^{\infty} r e^{-r(s-t)} \Psi_{s}^{\alpha^{*}} \mathrm{~d} B_{s}^{\alpha^{*}} \\
& =W_{t}^{\alpha^{*}}-\int_{t}^{\infty} r e^{-r(s-t)} g\left(\alpha_{s}^{*}\right) \mathrm{d} s+\int_{t}^{\infty} r e^{-r(s-t)} \Psi_{s}^{\alpha^{*}} \mathrm{~d} B_{s}^{\alpha} \\
& +\int_{t}^{\infty} r e^{-r(s-t)} \Psi_{s}^{\alpha^{*}}\left[f\left(\alpha_{s}\right)-f\left(\alpha_{s}^{*}\right)\right] \mathrm{d} s,
\end{aligned}
$$

and $\Psi_{t}^{\alpha^{*}}\left[f\left(\alpha_{t}\right)-f\left(\alpha_{t}^{*}\right)\right]=Z_{t}^{\alpha^{*}}\left[\mu\left(\alpha_{t}\right)-\mu\left(\alpha_{t}^{*}\right)\right]$, we get

$$
\begin{aligned}
W_{t}^{\alpha}-W_{t}^{\alpha^{*}} & =\mathrm{E}_{\mathcal{W}_{t}}^{\mathrm{P}_{t}}\left[\int_{t}^{\infty} r e^{-r(s-t)}\left[g\left(\alpha_{s}\right)+Z^{\alpha^{*}} \mu\left(\alpha_{s}\right)-g\left(\alpha_{s}^{*}\right)-Z_{s}^{\alpha^{*}} \mu\left(\alpha_{s}^{*}\right)\right] \mathrm{d} s\right. \\
& \left.+\int_{t}^{\infty} r e^{-r(s-t)}\left(\Psi_{s}^{\alpha^{*}}-\Psi_{s}^{\alpha}\right) \mathrm{d} B_{s}^{\alpha}\right] \\
& =\mathrm{E}_{\mathcal{W}_{t}}^{\mathrm{P}^{\alpha}}\left[\int_{t}^{\infty} r e^{-r(s-t)}\left[G\left(\alpha_{s}, Z_{s}^{\alpha^{*}}\right)-G\left(\alpha_{s}^{*}, Z_{s}^{\alpha^{*}}\right)\right] \mathrm{d} s\right]
\end{aligned}
$$


In particular, for the deviating player $i$, we conclude

$$
W_{t}^{i,\left(\alpha^{i}, \alpha^{-i, *}\right)}-W_{t}^{i, \alpha^{*}}=\mathrm{E}_{\mathcal{W}_{t}}^{\left.\mathrm{P}^{i}, \alpha^{-i, *}\right)}\left\{\int_{t}^{\infty} r e^{-r(s-t)}\left[G^{i}\left(\alpha_{s}, Z_{s}^{\alpha^{*}}\right)-G^{i}\left(\alpha_{s}^{*}, Z_{s}^{*}\right)\right] \mathrm{d} s\right\} \leq 0 .
$$

since $\alpha^{*}$ satisfies (19) by hypothesis. This implies that $W^{\alpha^{*}}$ is a perfect public equilibrium payoff process.

(ii) Suppose that $\alpha^{*}$ is a perfect public equilibrium strategy profile with corresponding continuation payoff process $W^{\alpha^{*}}$. Suppose that condition (19) is violated by the pair $\alpha^{*}, Z^{\alpha^{*}}$ for player $i$. Define the strategy

$$
\alpha_{t}^{i} \in \underset{a^{i} \in A^{i}}{\operatorname{argmax}} G^{i}\left(\left(a^{i}, \alpha_{t}^{-i, *}\right), Z_{t}^{\alpha^{*}}\right) .
$$

Since $A^{i}$ is finite and $Z_{t}^{\alpha^{*}}$ and $\alpha_{t}^{-i, *}$ are both $\mathcal{W}_{t}$-measurable, $\alpha_{t}^{i}$ is $\mathcal{W}_{t}$-measurable as well (if there are ties, then make some selection). Under the new public strategy profile $\left(\alpha^{i}, \alpha^{-i, *}\right)=\alpha$, the continuation payoff is

$$
W_{t}^{i, \alpha}=\mathrm{E}^{\mathrm{P}^{\alpha}}\left[\int_{t}^{\infty} r e^{-r(s-t)} g^{i}\left(\alpha_{s}\right) \mathrm{d} s \mid \mathcal{W}_{t}\right] .
$$

Mimicking the manipulation performed in part (i) of the proof, we see that

$$
\begin{aligned}
W_{t}^{i, \alpha}-W_{t}^{i, \alpha^{*}} & =\mathrm{E}_{\mathcal{W}_{t}}^{\mathrm{P}_{t}}\left[\int_{t}^{\infty} r e^{-r(s-t)}\left[g^{i}\left(\alpha_{s}^{i}, \alpha_{s}^{-i, *}\right)+Z^{i, \alpha^{*}} \mu\left(\alpha_{s}\right)-g\left(\alpha_{s}^{*}\right)-Z_{s}^{i, \alpha^{*}} \mu\left(\alpha_{s}^{*}\right)\right] \mathrm{d} s\right] \\
& =\mathrm{E}_{\mathcal{W}_{t}}^{\mathrm{P}_{t}}\left\{\int_{t}^{\infty} r e^{-r(s-t)}\left[G^{i}\left(\left(\alpha_{s}^{i}, \alpha_{s}^{-i, *}\right), Z_{s}^{\alpha^{*}}\right)-G^{i}\left(\alpha_{s}^{*}, Z_{s}^{\alpha^{*}}\right)\right] \mathrm{d} s\right\} \\
& >0
\end{aligned}
$$

where the last inequality follows from the hypothesis that the Nash-equilibrium condition (19) is violated. But this contradicts the initial hypothesis that $W^{\alpha^{*}}$ is a public perfect equilibrium payoff process.

\section{The open problem}

The previous sections have shown that continuation payoff processes in discrete as well as in continuous-time are characterized by orthogonal decompositions with respect to the driving martingale noise processes. Recall that in discrete time the continuation 
payoff process corresponding to a public strategy $\alpha^{h}$ is determined by the equation

$\left(\right.$ Cont $\left.^{h}\right) \quad W_{n+1}^{h}-W_{n}^{h}=\frac{1-\delta}{\delta}\left[W_{n}^{h}-G^{h}\left(\alpha_{n}^{h}, Z_{n+1}^{h}\right)\right]+r Z_{n+1}^{h} \Delta B_{n}^{h}+\delta^{-(n+1)} \Delta L_{n}^{h}$.

In continuous time the corresponding representation is

(Cont) $\quad \mathrm{d} W_{t}=r\left[W_{t}-G\left(\alpha_{t}, Z_{t}\right)\right] \mathrm{d} t+r Z_{t} \mathrm{~d} B_{t}+\mathrm{d} \eta_{t}$.

The open problem is to understand in which precise sense these two equations are similar. This is not at all an obvious question, but it is the key to understand the connection between repeated game dynamics in discrete and continuous time. Viewing these equations abstractly as BSDEs, a promising starting point might be to relate the repeated game dynamics with recent stability results on martingale representations reported in Jacod et al. (2000), and related results in the context of backward stochastic differential equations in Briand et al. (2002). The connection is however not very clear due to the presence of the control (the public strategies), which might lead to the chattering problem, which already is present in our weak convergence analysis (Staudigl and Steg, 2014). Still it is a central question for future research to solve this problem.

\section{References}

Abreu, D., D. Pearce, and E. Stacchetti (1990). Toward a theory of discounted repeated games with imperfect monitoring. Econometrica Vol. 58(5), 1041-1063.

Bain, A. and D. Crisan (2000). Fundamentals of Stochastic Filtering. Springer - Stochastic Modelling and Applied Probability.

Briand, P., B. Delyon, and J. Mémin (2002). On the robustness of backward stochastic differential equations. Stochastic Processes and their Applications 97(2), $229-253$.

Cox, J. C., S. A. Ross, and M. Rubinstein (1979). Option pricing: A simplified approach. Journal of Financial Economics 7(3), $229-263$.

Fujisaki, M., G. Kallianpur, and H. Kunita (1972). Stochastic differential equations for the non linear filtering problem. Osaka Journal of Mathematics Vol. 9, Issue 1, 19-40.

Jacod, J. (1979). Calcul stochastique et problèmes de martingales, Volume 714 of Lecture Notes in Mathematics. Spinger-Verlag.

Jacod, J., S. Meleard, and P. Protter (2000). Explicit form and robustness of martingale representations. The Annals of Probability 28(4), 1747-1780. 
Kunita, H. and S. Watanabe (1967). On square integrable martingales. Nagoya Mathematical Journal 30, 209-245.

Protter, P. E. (2005). Stochastic Integration and Differential Equations. Springer - Stochastic Modelling and Applied Probability.

Sannikov, Y. (2007). Games with imperfectly observable actions in continuous time. Econometrica 75(5), 1285-1329.

Staudigl, M. and J.-H. Steg (2014). On repeated games with imperfect public monitoring: From discrete to continuous time. Bielefeld University. 